\title{
Características epidemiológicas de la infección por Staphylococcus aureus meticilino resistente en el Hospital Clínico Regional de Valdivia
}

\author{
Mónica Gil D de $\mathbf{M}^{1}$, Carolina Cruz $\mathbf{P}^{1}$, Nora Leal $\mathbf{A}^{1}$, Laura Otth $\mathbf{R}^{2}$, María Emilia Arce $\mathbf{G}^{1}$,
} Angela Zaror $\mathbf{C}^{1}$ y Víctor Lizama $\mathbf{A}^{\mathbf{1}}$.

\section{RESUMEN}

Los factores de riesgo para adquirir una infección por Staphylococcus aureus meticilino resistente incluyen: factores del huésped, quirúrgicos y de la atención clínica. Dentro de los primeros están las edades extremas, estadía hospitalaria prolongada y enfermedades subyacentes como cáncer, diabetes e inmunosupresión. Los factores de la atención clínica involucran uso prolongado e inadecuado de antimicrobianos y múltiples procedimientos invasivos, entre otros. En el Hospital Clínico Regional de Valdivia, se realizó un análisis retrospectivo de 76 fichas clínicas de pacientes que tuvieron aislamientos de Staphylococcus aureus meticilino resistente en el transcurso de su hospitalización, en el período entre Enero y Junio de 1998. Estas cepas constituyeron el 60,8\% de las cepas aisladas en ese período. El aislamiento se efectuó en pacientes mayores de 40 años en el $84 \%$ de los casos y el $53,9 \%$ de los enfermos adquirió la bacteria posterior a los 10 días de hospitalización. La bacteria se aisló de la herida operatoria en un $27,6 \%$. Los factores de riesgo más frecuentes fueron el uso de antimicrobianos por más de 5 días antes del cultivo (76,3\%), cáncer $(27,6 \%)$ y enfermedad neurológica $(27,6 \%)$. El factor de riesgo de la atención clínica más frecuente fue la intervención quirúrgica (78,9\%). La infección nosocomial por Staphylococcus aureus meticilino resistente contribuye a aumentar significativamente la morbilidad, mortalidad y costo del hospital, por lo que es importante conocer la realidad local en relación a sus características epidemiológicas. (Palabras claves/Key words: Staphylococcus aureus meticilino resistente/Methicillin-resistant Staphylococcus aureus resistente; Infección intrahospitalaria/ Nosocomial infection; Factores de riesgo/Risk factors).

\section{INTRODUCCIÓN}

En 1961, Jevons ${ }^{1}$ reporta la primera cepa de Staphylococcus aureus meticilino resistente (SAMR) en Londres, destacándolo como una importante causa de infección nosocomial en Europa. En la siguiente década se extiende a Estados Unidos, provocando graves infecciones intrahospitalarias ${ }^{2}$, posteriormente se difunde a Japón y en la actualidad se aisla a nivel mundial ${ }^{3}$.

Es conocido su rol causal en las infecciones nosocomiales de grandes centros de salud ${ }^{4,5}$ y una vez establecidos en ellos es muy difícil de erradicar, lo que constituye una seria preocupación para los clínicos y salubristas.

Su incidencia aumenta progresivamente ${ }^{7}$ y está en directa relación con la exposición a procedimientos invasivos y a la transgresión de las normas de atención directa del paciente, básicamente el lavado de manos, fundamentado en el hecho de que el mecanismo de transmisión más importante es a través de la portación transitoria de las manos del personal del hospital ${ }^{8}$.

Nuestro hospital no escapa a la realidad de otros centros. Durante el año 1998 del total de infecciones intrahospitalarias, vigiladas por un 
sistema activo y selectivo (Sistema Nacional de Vigilancia Epidemiológica), el 19,9\% fue provocado por Staphylococcus aureus y de ellos el $51,8 \%$ era meticilino resistente. Al incluir la vigilancia de los cuadros infecciosos aumenta la prevalencia de Staphylococcus aureus a $27 \%$. Fue agente causal del $44,1 \%$ de las neumonías asociadas a ventilación mecánica, al $57,1 \%$ de las bacteremias en inmunodeprimidos y al $71,4 \%$ de las bacteremias asociadas a catéter de doble lumen. Los servicios más afectados en orden decreciente han sido permanentemente cirugía y medicina; este último constituye un servicio de riesgo al considerar que existe un $1,96 \%$ de portadores nasales de Staphylococcus aureus en el personal de este hospital, de los cuales cerca del $70 \%$ procede del servicio de medicina, representando el $41,6 \%$ de portación en ese servicio.

Otro aspecto fundamental son las características propias de los pacientes, siendo los más afectados aquellos con patología de base ${ }^{10}$ que utilizan antimicrobianos de amplio espectro y tienen una estadía hospitalaria prolongada ${ }^{11}$.

En el presente trabajo hemos querido recopilar los antecedentes de aquellos pacientes que han tenido aislamientos de SAMR durante su hospitalización, con el propósito de conocer las características del huésped y los factores de riesgo asociados, contribuyendo de esa manera al conocimiento de lo que ocurre en nuestro medio y enfatizar las medidas preventivas frente al paciente que constituye grupo de riesgo.

\section{MATERIAL Y MÉTODOS}

Se realizó un análisis retrospectivo de 76 fichas clínicas, correspondientes a pacientes que tuvieron al menos un aislamiento de SAMR en el transcurso de su hospitalización, en el período de Enero a Junio de 1998.

El diagnóstico microbiológico y estudio de sensibilidad antimicrobiana fue realizado a través de método semiautomatizado "Sensident", en la sección de bacteriología del Laboratorio Central del Hospital Clínico Regional de Valdivia.

El análisis estadístico incluyó tamaño muestral con un nivel de confianza de $95 \%$ y precisión de $5 \%$, con una prevalencia de factores de riesgo de $82 \%$, lo que da un $n=76$.

En la tabla 6 se incluyó el método $F$ de Fisher $=0,69$, con un $p>0,05$, lo que permite inferir que la asociación entre estas dos variables no es estadísticamente significativa.

\section{RESULTADOS}

Los Servicios de procedencia de las cepas de SAMR se detallan en la Tabla 1 y corresponden al $60,68 \%$ de las cepas aisladas durante ese período.

\section{Tabla 1. Servicios clínicos de procedencia de las cepas de Staphylococcus aureus meticilino resistente.}

\begin{tabular}{|lcl|}
\hline & SAMR & \\
Servicio & $\mathbf{N} / \mathbf{n}$ & $\%$ \\
\hline Cirugía & $33 / 60$ & 55 \\
Medicina & $17 / 25$ & 68 \\
Traumatología & $12 / 14$ & 85,7 \\
Neurocirugía & $10 / 13$ & 76,9 \\
Urología & $5 / 5$ & 80 \\
\hline Total & $76 / 117$ & 64,9 \\
\hline
\end{tabular}

№: número de cepas de SAMR a los cuales se les hizo revisión de ficha clínica.

no: total de cepas de SAMR aisladas primer semestre 1998. $\mathrm{P}<0,05$.

De los 76 casos de aislamiento de SAMR en nuestro estudio, el $65,8 \%$ fue en el sexo masculino, principalmente en el grupo etáreo de 61 a 80 años (46\%), siendo el $84 \%$ de los pacientes con edad sobre los 40 años (Tabla 2).

Tabla 2. Distribución de Staphylococcus aureus meticilino resistente en 76 pacientes según sexo y edad.

\begin{tabular}{|llrl|}
\hline & & № & $\%$ \\
\hline \multirow{2}{*}{ Sexo } & Femenino & 26 & 34,2 \\
& Masculino & 50 & 65,8 \\
\hline \multirow{5}{*}{ Edad } & $<20$ años & 2 & 2,63 \\
& $20-40$ años & 10 & 13,15 \\
& $41-60$ años & 22 & 28,9 \\
& $>81-80$ años & 35 & 46 \\
& $>80$ años & 7 & 9,2 \\
\hline
\end{tabular}

La Tabla 3 nos muestra que alrededor de la mitad de los casos $(53,9 \%)$ adquirieron la bacteria posterior a los diez días de hospitalización. Destaca que 18 del total de los pacientes $(23,7 \%)$ ingresaron 
Tabla 3. Número de días de hospitalización previo al cultivo en relación a procedencia y hospitalización anterior del paciente.

\begin{tabular}{|lcccccccc|}
\hline $\begin{array}{l}\text { Días de } \\
\text { hospitalización } \\
\text { previo al cultivo }\end{array}$ & & Procedencia & \multicolumn{3}{c}{$\begin{array}{c}\text { Hospitalización anterior } \\
\text { último año }\end{array}$} & Total \\
& Comunidad & Otro servicio & Otro hospital & Sí & No & No & $\%$ \\
\hline$<2$ días & 16 & 1 & 1 & 16 & 2 & 18 & 23,7 \\
$2-5$ días & 5 & 1 & 2 & 4 & 4 & 8 & 10,5 \\
$5-10$ días & 7 & 1 & 1 & 4 & 5 & 9 & 11,8 \\
$>10$ días & 23 & 10 & 8 & 10 & 31 & 41 & 53,9 \\
\hline Total & 51 & 13 & 12 & 34 & 42 & 76 & 100 \\
& $(67,1 \%)$ & $(17,1 \%)$ & $(15,8 \%)$ & $(44,7 \%)$ & $(55 \%)$ & & \\
\hline
\end{tabular}

al hospital colonizados o infectados (cultivo positivo antes de las $48 \mathrm{~h}$ de hospitalización), asociado a su vez a mayor incidencia de hospitalizaciones repetidas el último año. El $67,1 \%$ procede de la comunidad, y un porcentaje menor proviene de otro servicio u hospital $(17,1 \%$ y $15,8 \%$ respectivamente).

Staphylococcus aureus meticilino resistente se aisló preferentemente de herida operatoria $(27,6 \%)$, seguido de cultivo de secreción de diferentes orígenes (Tabla 4).

Los factores de riesgo más frecuentemente encontrados en el huésped fue el uso de antimicrobianos de amplio espectro, bi o triasociados por período mayor a cinco días antes del cultivo $(76,3 \%)$, además de enfermedad neoplásica y neurológica en un $27,6 \%$ cada uno (Tabla 5).

En relación a los factores de riesgo de la atención clínica, el 78,9\% de los pacientes habían sido sometidos a algún tipo de intervención quirúrgica, el 44,7\% usaron catéter venoso periférico y el $35,5 \%$ estaban con catéter urinario permanente. La Tabla 6 muestra que el $81,5 \%$ y el $97,3 \%$ de los pacientes tenían factores atribuibles al huésped y a la atención clínica respectivamente y en el $78,9 \%$ de los casos existían ambos antecedentes de riesgo. No hubo paciente sin factor de riesgo.

\section{DISCUSIÓN}

Staphylococcus aureus meticilino resistente como patógeno nosocomial ha incrementado su incidencia desde 2,4\% en 1975 al 29\% en 1991 en países desarrollados ${ }^{12}$. En Chile se ha
Tabla 4. Sitio de aislamiento de Staphylococcus aureus meticilino resistente I semestre 1998.

\begin{tabular}{|lrr|}
\hline Procedencia de la muestra & № & $\%$ \\
\hline Herida operatoria & 21 & 27,6 \\
- limpia & 2 & \\
- limpia contaminada & 8 & \\
- contaminada & 3 & \\
- sucia & 8 & \\
Escaras & 14 & 18,4 \\
Hemocultivos & 5 & 6,6 \\
Líquidos estériles & 5 & 6,6 \\
$\quad$ - peritoneal & 2 & \\
- LCR & 1 & \\
- articular & 1 & \\
- pleural & 1 & \\
Orina & 6 & 7,9 \\
Secreción bronquial & 3 & 3,9 \\
Otras secreciones & 22 & 28,9 \\
\hline Total & 76 & 100 \\
\hline
\end{tabular}

comunicado un $22 \%$ en 1991 y un $33 \%$ en $1995^{13}$. En el ambiente hospitalario afecta a un determinado tipo de pacientes, incluyendo a aquellos huéspedes con factores predisponentes, lo que explica la mayor incidencia en edades extremas, donde la inmadurez propia del recién nacido o el deterioro fisiológico del sistema inmune del anciano facilita la ocurrencia de infección.

En la literatura médica se describe el aislamiento ocasional de este microorganismo en 
Tabla 5. Distribución de los factores de riesgo asociados.

\begin{tabular}{|llrc|}
\hline Factor de riesgo & Tipo & $\begin{array}{c}\text { \% en relación al total } \\
\text { de pacientes (76) }\end{array}$ \\
\hline & Uso de antibióticos previos & 58 & 76,3 \\
& Neoplasia & 21 & 27,6 \\
& Enfermedad neurológica & 21 & 27,6 \\
& Hipertensión arterial & 14 & 18,4 \\
& Diabetes mellitus & 13 & 17,1 \\
Del huésped & Insuficiencia renal & 8 & 10,5 \\
& Cardiopatía & 7 & 9,2 \\
& Desnutrición & 5 & 6,6 \\
& Inmunodepresión & 3 & 3,9 \\
& Quemadura & 2 & 2,6 \\
& Otro & 7 & 9,2 \\
\hline \multirow{5}{*}{ De la atención clínica } & 60 & 78,9 \\
& Intervención quirúrgica & 34 & 44,7 \\
& Catéter venoso periférico & 27 & 35,5 \\
& Catéter urinario permanente & 14 & 18,4 \\
& Catéter venoso central & 6 & 7,9 \\
& Ventilación mecánica & 5 & 6,6 \\
& Diálisis & 2 & 2,6 \\
& Nutrición parenteral & 14 & 18,4 \\
\hline
\end{tabular}

Tabla 6. Relación entre factores de riesgo del huésped y de la atención clínica.

\begin{tabular}{|ccccc|}
\hline Factores de riesgo del huésped & \multicolumn{2}{c}{ Factores de riesgo de la atención clínica } & \multicolumn{2}{c|}{ Total } \\
& SI & NO & № & $\%$ \\
\hline SI & 60 & 2 & 62 & 81,5 \\
NO & 14 & 0 & 14 & 18,4 \\
\hline Total & 74 & 2 & 76 & 100 \\
& $(97,3 \%)$ & $(2,7 \%)$ & & \\
\hline
\end{tabular}

$\mathrm{F}$ de Fisher $=0,69$

$p>0,05$

el paciente ambulatorio ${ }^{14}$, lo que no concuerda con lo encontrado en nuestra revisión, en que el $67,1 \%$ del grupo estudiado procedían de la comunidad, hecho que se explica si se considera que son pacientes con múltiples controles en policlínico y hospitalizaciones reiteradas por patologías crónicas o agudas parcialmente resueltas; en contraste con los 23 pacientes que adquirieron la bacteria después de una prolongada estadía hospitalaria sin que hallan estado previamente en el hospital. Si se considera el grupo de pacientes ambulatorios que nunca han estado hospitalizado previamente, éste correspondería solamente al 2,6\% (2/73).
Es conocido el hecho de que un mayor contacto con la flora nosocomial y con el personal de salud portador permanente o transitorio de SAMR por períodos prolongados, tiene un valor predictivo para el desarrollo de la infección. National Nosocomial Infection Study (NISS) dispone de una base de datos para estratificar a los pacientes en relación al riesgo de infección, incluyendo procedimiento quirúrgico mayor de dos horas, intervenciones intraabdominales y presencia de 3 o más factores de riesgo previamente establecidos ${ }^{15}$. Los factores del huésped que han sido propuestos incluyen la edad, la obesidad mórbida, desnutrición, diabetes 
mellitus, cáncer, inmunodepresión, enfermedad severa e infección a distancia ${ }^{16}$, similar a los factores predisponentes que favorecen la infección por otro tipo de agentes nosocomiales. Esto concuerda ampliamente con lo encontrado en nuestro estudio en el cual la mayoría de los pacientes tenían neoplasia $(27,6 \%)$, enfermedad neurológica, principalmente paraplejia $(27,6 \%)$, hipertensión arterial y diabetes. Cabe mencionar que otro factor que contribuye a la infección es el uso de antibióticos de amplio espectro ${ }^{16}$, antecedente que logramos corroborar en el $76,3 \%$ de los pacientes.

Existen factores de la atención clínica como la exposición a procedimientos quirúrgicos o instrumentalización ${ }^{16}$. Dentro de este aspecto se incluye como riesgoso la duración prolongada de la cirugía, contaminación intraoperatoria, hemostasia deficiente, cuerpo extraño y espacio muerto, entre otros, que lleva a la entrada del microorganismo al sitio quirúrgico por disrupción del tejido y que se ve favorecido con la susceptibilidad intrínseca del huésped a la infección.

Esto explica el alto número de pacientes portadores de la bacteria que previamente habían sido intervenidos $(78,9 \%)$. De esta manera los pacientes más afectados son aquellos hospitalizados en los servicios de UCl, Unidad de Quemados, Cirugía y Neonatología ${ }^{17}$.

A futuro, no sólo se debiera identificar al paciente con probabilidad incrementada de infección por SAMR, sino también desarrollar indicadores de riesgo sensibles y específicos con buen poder predictivo y debidamente validados para disminuir la morbimortalidad y el gasto creciente en salud.

\section{REFERENCIAS}

1. Jevons MP: Celbenin-resistant Staphylococci. Br Med J 1961; 1: 124-5

2. Klimek JJ, Marskik FJ, Bartlett RC et al: Clinical, epidemiologic and bacteriologic observations of an outbreak of methicillin-resistant Staphylococcus aureus at a large community hospital. Am J Med 1976; 61: 340-5

3. Pascual A. Staphylococcus. Microbiología Clínica 1990; 39: 575-85

4. Barrett FF, Mc Ghehee RP, Finland M: Methicillinresistant Staphylococcus aureus at Boston City Hospital. Bacteriologic and epidemiologic observations. N Engl J Med 1968; 279: 441-8

5. Boyce JM, White RL, Spruill EY: Impact of methicillin-resistant Staphylococcus aureus on the incidence of nosocomial infections. J Infect Dis 1983; 148: 763

6. Parras F, Rodríguez Creixems $\mathrm{M}$, Muñoz $\mathrm{P}$ et al: Brote epidémico de Staphylococcus aureus resistente a meticilina (SARM) en un hospital general. Informe Preliminar. Enf Infec Microbiol Clin 1991; 9: 200-7

7. Pérez Trallero E, García Avenzara JM: Prevalence of methicillin-resistant Staphylococcus aureus in a spanish hospital. Rev Infect Dis 1988; 10: 627-8

8. Thompson RL, Cabezudo J, Wenzel RP: Epidemiology of nosocomial infections caused by methicillin- resistant Staphylococcus aureus. Ann Intern Med 1982; 97: 309-17

9. Gil M: Tesis: Estudio de portadores nasales de Staphylococcus aureus sensibles y resistentes a meticilina en personal del Hospital Clínico Regional Valdivia, 1996

10. Perl TM, Roy MC: Postoperative wound infections: Risk factors and role of Staphylococcus aureus nasal carriage. J Chemother 1995; 7 Suppl 3: 29-35

11. Leape LL, Brennan TA, Laird N et al: The nature adverse events in hospitalized patients: result of the Harvard Medical Practice Study II. N Engl J Med 1991; 324: 377-84

12. Emori TG, Gaines RP: An overview of nosocomial infections, including the role of the microbiology laboratory. Clin Microbiol Rev 1993; 6: 428-42

13. Fernández A, Pinto ME, Worstman C: Bacteremia intrahospitalaria: evaluación de cinco años. Rev Chil Infect1991; 8: 158-61

14. Garibaldi RA, Cushing D, Leres T: Risk factor for postoperative infection. Am J Med 1991; 91(3B): 158S-163S.

15. Slaughter MS, Olson MM, Lee JT, Ward HB: A fifteen year wound surveillance study after coronary artery by pass. Ann Thorac Surg 1993; 56: $1063-8$

16. Pitter D, Ducel G: Infectious risk factors related operating rooms. Infect Control Hosp Epidemiol 1994; 15: 456-62

17. Boyce JM: Methicillin resistant Staphylococcus aureus in hospitals and long term care facilities: microbiology, epidemiology and preventive measures. Infect Control Hosp Epidemiol 1992; 13: $725-37$ 